\title{
Analysis of Cavitation Processes in Xylem
}

\author{
Fanyi Shen \\ College of Science, Beijing Forestry University, Beijing, China \\ Email: fyshen@bjfu.edu.cn
}

How to cite this paper: Shen, F.Y. (2020) Analysis of Cavitation Processes in Xylem. Journal of Applied Mathematics and Phys ics, 8, 1767-1778. https://doi.org/10.4236/jamp.2020.89133

Received: May 25, 2020

Accepted: September 11, 2020

Published: September 14, 2020

Copyright (c) 2020 by author(s) and Scientific Research Publishing Inc. This work is licensed under the Creative Commons Attribution International License (CC BY 4.0).

http://creativecommons.org/licenses/by/4.0/

\begin{abstract}
Cavitation in plants is caused by development of air bubbles, which is related to their equilibrium and development. There is a univariate cubic equation for bubble balance. New root formula of this kind of equation was proposed by Shenjin Fan, which is simpler than the Caldan's. Using Shenjin formulas and taking water pressure $P_{1}$ as an independent variable, this paper gives the exact solution of the equation under certain conditions. The stability of the equilibrium of an air bubble in its different radius ranges is obtained by the way different from the previous. This kind of cavitation includes two types: First type may be caused by the growth of pre-existent air bubbles; Second type is air seeding, here defined as the sucking of air bubbles from already gas-filled conduits. For air seeding three ways of cavitation have been proposed. For the first type this paper puts forward that two ways of cavitation can occur, which are the same with the first two ways of air seeding except of air reservoirs. Moreover, for the first way of the first type, the range of water pressures is the same with that of the first way of air seeding. For the second way of the first type the range of water pressures is much wider, or the pressure range equals the pressure sum of the second and third ways of air seeding. Through the specific data the relationship between the two types is given.
\end{abstract}

\section{Keywords}

Bubble, Cavitation, Growing of Air Bubbles, Air Seeding, Xylem Pressure

\section{Introduction}

Water ascends plants in a metastable state under tension, meaning that the xylem sap pressure is more negative than that of the vapor pressure of water [1]. This is a physical effect. In the process cavitation, which is defined as void formation in xylem sap, often occurs in conduits [2]. Cavitation of water columns within the xylem is deleterious for plant water relations because it results in embolism thereby reducing hydraulic conductivity [3]. Cavitation is induced by air 
seeding, which can occur when air passes through the pores of pit membranes to form bubbles [1] [4]. Cavitation is also caused by tiny air bubbles adhering to cracks in vessel walls [1]. Zimmermann [5] studied the process of air seeding. He concluded that once a bubble formed by air seeding enters into a vessel, it immediately expands until the tension forces on the wall are released.

Nowadays some researchers pay their attentions to the facts: angiosperm xylem contains abundant hydrophobic surfaces; there are insoluble surfactants in xylem and bordered pit membranes play a crucial role in drought-induced embolism formation via the process of air-seeding etc. [6] [7]. Whether the capillary failure is an appropriate physical model comes to be a question [7]. Then, from the experiments [8] [9] [10] [11], it is obvious that the former hypothesis of air seeding is still effective to the xylem of some trees although cavitation in lipid bilayers has negative pressure stability limit [12].

Where does an air seeding event take place? Considering the potential importance of the rare pit hypothesis, Plavcová et al. [13] suggested that more attention should be paid to the structural irregularities, as those may represent the rare sites ultimately responsible for air-seeding.

Isolated conduit has been seen, which might be caused by another mechanism [9] [11]. The development of nanobubbles snapped off at pit membranes can also cause cavitation events [14]. These all may involve the growth of pre-existent air bubbles in xylem.

Ponomarenko et al. [11] distinguished two types of optical events. The first is the "nucleation" events, starting in a fully wet area, which might be caused by the growth of pre-existent air bubbles. The second is the "air-seeding" events, being defined as the appearance of bubbles near an already gas-filled conduit. The definition of types of cavitation in this paper follows that defined by Ponomarenko et al. [11].

Three ways of cavitation by air seeding have been proposed [15] (In the article [15] the word "way" was defined as word "type"). The types of cavitation by the growth of pre-existent air bubbles in xylem should be given more attention.

The two types of cavitation are all related to the equilibrium, stability and development of air bubbles in xylem. Analysis of bubble expansion by mechanism and by the equilibrium criterion of Helmholtz function has been made, which is based on the equation of bubble balance [16] [17]. This is a univariate cubic equation. Taking mole number $n$ of air in a bubble as an independent variable, its analytic solution has been made [18]. A new formula for finding the root of univariate cubic equation was proposed by Fan [19], which is simpler than the Caldan's. Using Shenjin formula and taking absolute water pressure $P_{1}$ as an independent variable, this paper gives the exact solution of the equation of bubble balance under certain conditions. As gas super-saturation is likely to occur in xylem sap almost daily [14], here the number $n$ is regarded as a constant. And the stability of the equilibrium of an air bubble in its different radius ranges is obtained by the way different from our previous article [17]. 
For the first type this paper puts forward two ways of cavitation, which are the same with the first two ways of air seeding except of air reservoirs, etc. Then, the relationship of the two types of cavitation is given.

\section{Air Babbles in Xylem}

\subsection{Equilibrium Equation of Air Bubbles}

Provided there is a bubble of radius $r$ with $n$ mole air in xylem sap. In order to simplifying the problem, several assumptions were made. First, because the water saturation vapor pressure in a bubble is generally less than $0.0023 \mathrm{MPa}$ at $20^{\circ} \mathrm{C}$, comparing with atmospheric pressure $P_{0}$, it is ignored. We also ignore some facts, including abundant hydrophobic surfaces and insoluble surfactants in xylem.

According to the ideal gas law $P=n R T / V_{\mathrm{g}}$, the gas pressure $P$ of the bubble of volume $V_{\mathrm{g}}=\left(4 \pi r^{3}\right) / 3$ should be

$$
P=3 n R T /\left(4 \pi r^{3}\right)
$$

When a bubble is in an equilibrium, we have:

$$
P_{\mathrm{g}}=P_{1}+2 \sigma / r
$$

The relationship among $P_{1}$, atmospheric pressure $P_{0}$ and xylem pressure $P_{1}^{\prime}$ is $P_{1}=P_{0}+P_{1}^{\prime}$. The point of intersection of the two curves $P(r)$ and $P_{\mathrm{g}}(r)$, or at $P=P_{\mathrm{g}}$, indicates temporary equilibrium of a bubble (Figure 1 ).

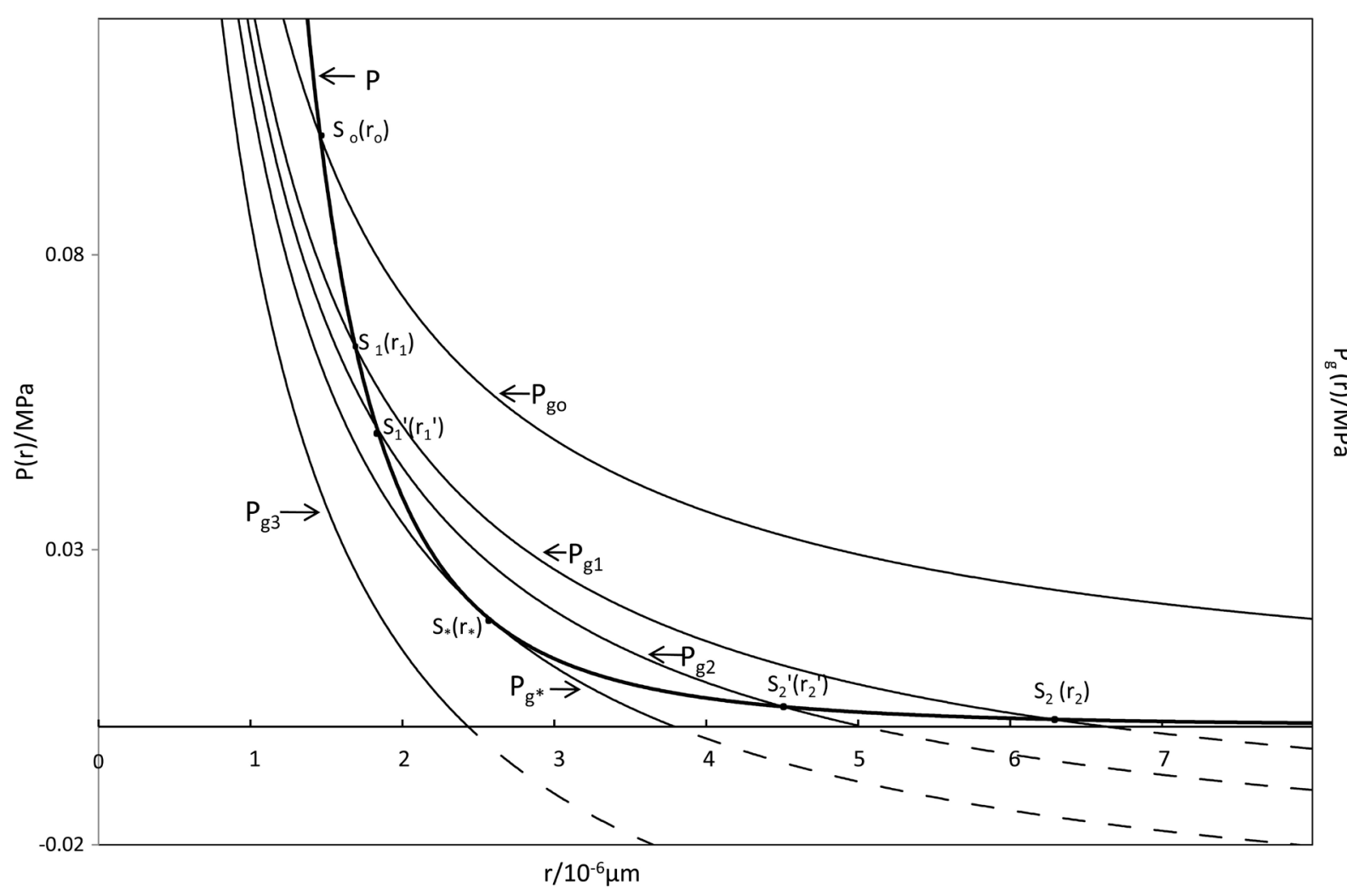

Figure 1. $P(r)$ and $P_{\mathrm{g}}(r)$ at different values of $P_{1}$. 
Thus, the positive real roots of the following equation

$$
4 \pi P_{1} r^{3}+8 \pi \sigma r^{2}-3 n R T=0
$$

is the radii of the bubble in an equilibrium.

Above system consists of three parts: an air bubble, the surrounding water and the interface between the air and the water. Corresponding to a fluctuation, the changes of its Helmholtz function are: $\mathrm{d} F_{\mathrm{g}}=-P \mathrm{~d} V_{\mathrm{g}}$ for the air, $\mathrm{d} F_{1}=P_{1} \mathrm{~d} V_{\mathrm{g}}$ for the water, and $\mathrm{d} F_{\mathrm{s}}=\sigma \mathrm{d} A$ for the increase of the gas/water interface $\mathrm{d} A$ $\left(A=4 \pi r^{2}\right)$. Thus, the total change of the Helmholtz function $\mathrm{d} F$ is $\mathrm{d} F=-(3 n R T / r) \mathrm{d} r+P_{1} 4 \pi r^{2} \mathrm{~d} r+\sigma 8 \pi r \mathrm{~d} r$, or

$$
F^{\prime}(r)=\mathrm{d} F / \mathrm{d} r=4 \pi P_{1} r^{2}+8 \pi \sigma r-3 n R T / r
$$

Integrating Expression (4) gives

$$
F(r)=\left(4 \pi P_{1} r^{3}\right) / 3+4 \pi \sigma r^{2}-3 n R T \ln r+C
$$

Once Helmholtz function $F(r)$ (Figure 2) reaches an extreme, or $F^{\prime}(r)=0$, the bubble will attain its equilibrium. Thus, from expression 4 we also have Equation (3).

\subsection{Solution of Equation (3)}

Letting the left side of Equation (3) be a function of $r$ gives

$$
f(r)=4 \pi P_{1} r^{3}+8 \pi \sigma r^{2}-3 n R T
$$

and

$$
f^{\prime}(r)=12 \pi P_{1} r^{2}+16 \pi \sigma r
$$

Therefore, the real roots of Equation (3) are the intersections of the curve $f(r)$ with $r$-axis and those the abscissa values of which are more than zero are the radii of the bubble in equilibrium (Figure 3 ).

1) When $P_{1}=0, f\left(r_{\mathrm{o}}\right)=8 \pi \sigma r_{\mathrm{o}}^{2}-3 n R T=0 \rightarrow r_{\mathrm{o}}=\frac{\sqrt{3}}{2} \sqrt{\frac{n R T}{2 \pi \sigma}}$.

2) If $P_{1} \neq 0$, corresponding to the following equation

$$
a x^{3}+b x^{2}+c x+d=0
$$

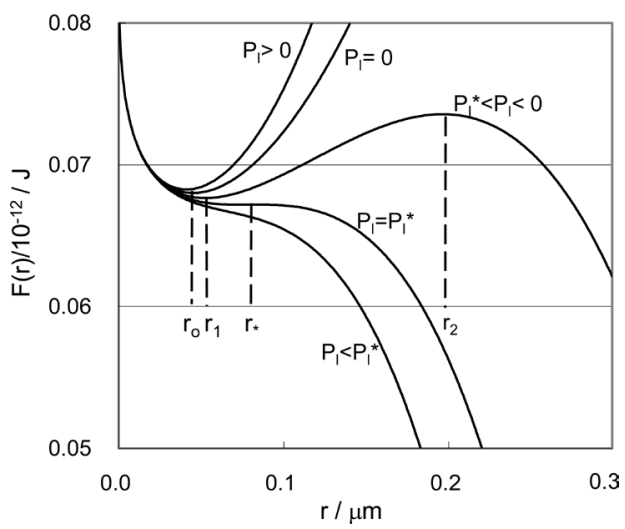

Figure 2. Graphs of Helmholtz function $F(r)$. 


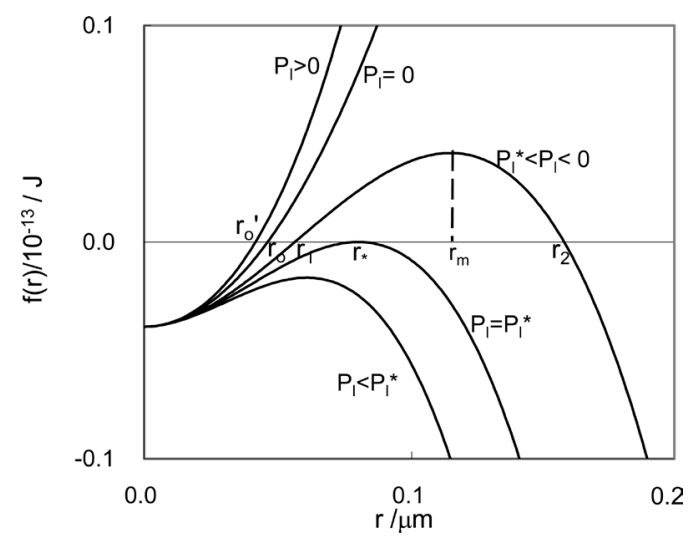

Figure 3. Graphs of $f(r)$.

the analytic solution of Equation (3) can be gotten by Shenkin formula [19].

For Equation (3), we obtained

$A=b^{2}-3 a c=(8 \pi \sigma)^{2}, \quad B=b c-9 a d=3 \times 4 \times 9 \pi P_{1} n R T$ and $C=c^{2}-3 b d=9 \times 8 \pi \sigma n R T$. Then, we got

$$
\Delta=B^{2}-4 A C=3^{2} \times 4^{2} \pi^{2} \times n R T\left(81 P_{1}^{2} n R T-128 \pi \sigma^{3}\right)
$$

If $\Delta=0,81 P_{1}^{2} n R T=128 \pi \sigma^{3}$, or $P_{1}^{*}= \pm \frac{8 \pi \sigma}{9} \sqrt{\frac{2 \pi \sigma}{n R T}}$. From Shenjin formula (3) [19], $k=\frac{B}{A}=\frac{8 \sigma}{3 P_{1}^{*}}, \quad x_{1}=-\frac{b}{a}+k=r_{1}=\frac{2 \sigma}{3 P_{1}^{*}}, \quad x_{2}=x_{3}=-\frac{k}{2}=r_{2}=r_{3}=-\frac{4 \sigma}{3 P_{1}^{*}}$. When $P_{1}^{*}>0, r_{1}>0, \quad r_{2}=r_{3}<0$. When $P_{1}^{*}<0, \quad r_{1}<0 \quad$ and $r_{2}=r_{3}=-\frac{4 \sigma}{3 P_{1}^{*}}=\frac{3}{2} \sqrt{\frac{n R T}{2 \pi \sigma}}=r_{*}>0$. By other ways, both $P_{1}^{*}=-\frac{8 \pi \sigma}{9} \sqrt{\frac{2 \pi \sigma}{n R T}}$ and $r_{*}=\frac{3}{2} \sqrt{\frac{n R T}{2 \pi \sigma}}\left(P_{1}^{*}=-4 \sigma / 3 r_{*}\right)$ have been gotten [16] [17] [20] [21] except of considering water vapor pressure $P_{\mathrm{v}}$ in the articles [20] [21]. Thus, $128 \pi \sigma^{3}$ can be replaced by $81\left(P_{1}^{*}\right)^{2} n R T$.

(1) When $0<P_{1}<P_{0}$, using Shenjin formula (2) and (4) [19], the analytical solution $r_{\mathrm{o}}^{\prime}>0$ of $f(r)=0$ can been obtained, knowing that $r_{0}^{\prime}<\frac{\sqrt{3}}{2} \sqrt{\frac{n R T}{2 \pi \sigma}}=r_{0}$. As xylem pressure $P_{1}$ is often negative, we do not pay more attention to it.

(2) When $P_{1}<0$ there are several situations as follows.

a) If $\Delta<0$, meaning $81 P_{1}^{2} n R T<81\left(P_{1}^{*}\right)^{2} n R T$, or $P_{1}^{*}<P_{1}<0$, from Shenjin formula (4) [19], there are three real roots for Equation (3). Letting $T=\frac{2 A b-3 a B}{2 \sqrt{A^{3}}}$ in Shenjin formula (4) [19]. By calculating, $T=1-\frac{2 P_{1}^{2}}{\left(P_{1}^{*}\right)^{2}}$ and $\theta=\operatorname{arecoc}\left(1-\frac{2 P_{1}^{2}}{\left(P_{1}^{*}\right)^{2}}\right)$. Then we obtained the solutions of Equation (3) as fol- 
lows.

i) $r_{\mathrm{I}}=-\frac{2 \sigma}{3 p_{1}}\left(1+2 \cos \frac{\theta}{3}\right)$. When $P_{1}=P_{1}^{*} \rightarrow T=-1$ and $\theta=\pi$, then $r_{\mathrm{I}}=-\frac{4 \sigma}{3 P_{1}^{*}}=\frac{3}{2} \sqrt{\frac{n R T}{2 \pi \sigma}}=r_{*}$. When $P_{1} \rightarrow 0, r_{\mathrm{I}} \rightarrow \infty$. Therefore, $r_{\mathrm{I}}$ is $r_{2}$ in Figure 3 , the values of which are in the range of $r_{*} \leq r_{2}<\infty$.

ii) $r_{\mathrm{II}}=-\frac{2 \sigma}{3 P_{1}}\left[1-2 \sin \left(\frac{\pi}{6}+\frac{\theta}{3}\right)\right]$. When $P_{1}=P_{1}^{*}, \quad r_{\mathrm{II}}=\frac{2 \sigma}{3 P_{1}^{*}}$. If $P_{1} \rightarrow 0$, $r_{\text {II }} \rightarrow \frac{8 \sigma}{9} \times \frac{\sqrt{3}}{2 P_{1}^{*}}$. The values of $r_{\text {II }}$ all are negative and should not be considered. iii) $r_{\text {III }}=-\frac{2 \sigma}{3 P_{1}}\left[1-2 \sin \left(\frac{\pi}{6}-\frac{\theta}{3}\right)\right]$. When $P_{1}=P_{1}^{*}$, we got $r_{\text {III }}=-\frac{4 \sigma}{3 P_{1}^{*}}=\frac{3}{2} \sqrt{\frac{n R T}{2 \pi \sigma}}=r_{*}$. While $P_{1} \rightarrow 0, \quad r_{\text {III }} \rightarrow-\frac{8 \sigma}{9} \times \frac{\sqrt{3}}{2 P_{1}^{*}} \rightarrow \frac{\sqrt{3}}{2} \sqrt{\frac{n R T}{2 \pi \sigma}}=r_{0}$. Thus, $r_{\mathrm{III}}$ is $r_{1}$ in Figure 3, the values of which are in the range of $r_{\mathrm{o}}<r_{1} \leq r_{*}$.

Therefore, in the range of $P_{1}^{*}<P_{1}<0$,

$$
\begin{gathered}
r_{1}=-\frac{2 \sigma}{3 P_{1}}\left[1-2 \sin \left(\frac{\pi}{6}-\frac{1}{3} \arccos \left(1-\frac{2 P_{1}^{2}}{\left(P_{1}^{*}\right)^{2}}\right)\right)\right] \quad\left(r_{\mathrm{o}}<r_{1}<r_{*}\right) \\
r_{2}=-\frac{2 \sigma}{3 P_{1}}\left(1+2 \cos \frac{1}{3} \arccos \left(1-\frac{2 P_{1}^{2}}{\left(P_{1}^{*}\right)^{2}}\right)\right) \quad\left(r_{*}<r_{2}<\infty\right)
\end{gathered}
$$

are the solutions of Equation (3), which are none than the formulas 10 in the article [18] except $\frac{n}{n_{\text {crit }}}$ being replaced by $\frac{P_{1}^{2}}{\left(P_{1}^{*}\right)^{2}}\left(n_{\text {crit }}\right.$ is the maximum mole number of the gas, which a bubble could contain at its $\left.P_{1}^{*}\right)$. During the decreasing of $P_{1}$, the values of $r_{1}$ and $r_{2}$ will get close to each other gradually to merge into $r_{*}$ at $P_{1}=P_{1}^{*}$ (Figure 1).

b) When $\Delta>0$, meaning $81 P_{1}^{2} n R T>81\left(P_{1}^{*}\right)^{2} n R T$, or $P_{1}<-\frac{8 \pi \sigma}{9} \sqrt{\frac{2 \pi \sigma}{n R T}}=P_{1}^{*}$, according to Shenjin formula (2) [19] there are a conjugate pair of complex roots and one real root for Equation (3). The real root is negative. This means that a gas bubble could not be in any equilibrium when $P_{1}<-\frac{8 \pi \sigma}{9} \sqrt{\frac{2 \pi \sigma}{n R T}}=P_{1}^{*}$.

To sum up, if $0<P_{1}<P_{0}$, Equation (3) has a positive real root $r_{0}^{\prime}$; If $P_{1}=0$, its positive real solution is $r_{\mathrm{o}}=\frac{\sqrt{3}}{2} \sqrt{\frac{n R T}{2 \pi \sigma}}\left(r_{\mathrm{o}}^{\prime}<r_{\mathrm{o}}\right)$; If $P_{1}=P_{1}^{*}=-\frac{8 \pi \sigma}{9} \sqrt{\frac{2 \pi \sigma}{n R T}}$, the positive real root is $r_{*}=\frac{3}{2} \sqrt{\frac{n R T}{2 \pi \sigma}}$. In the range of $P_{1}^{*}<P_{1}<0$, the positive real roots of Equation (3) are Formula (8). The relationship of radii of an air 
bubble is $r_{0}^{\prime}<r_{\mathrm{o}}<r_{1}<r_{*}<r_{2}$.

\subsection{Stability of Bubble Equilibrium}

The stability of an air bubble which is in equilibrium depends on Formula (6b). For $f(r)=0$, if $f^{\prime}(r)>0, F(r)$ reaches its minimum and the equilibrium of the bubble is stable. In turn, $f^{\prime}(r)<0, F(r)$ arrives at its maximum, the equilibrium of the bubble is unstable.

1) When $0 \leq P_{1}<P_{0}$, formula $f^{\prime}(r)>0$ for all $r>0 . F\left(r_{\mathrm{o}}\right)$ and $F\left(r_{\mathrm{o}}^{\prime}\right)$ are the minima and the bubbles of radius $r_{0}$ or $r_{0}^{\prime}$ in xylem are stable.

2) When $P_{1}^{*}<P_{1}<0$, there are two roots $r_{1}$ and $r_{2}\left(r_{2}>r_{1}\right)$ for Equation (3). When $f^{\prime}\left(r_{\mathrm{m}}\right)=0$, we have $r_{\mathrm{m}}=-\frac{4 \sigma}{3 P_{1}}$. Thus, $f(r)$ reaches its extremum at $r_{\mathrm{m}}$ and $r_{1}<r_{\mathrm{m}}<r_{2}$ (Figure 3).

For $r_{1}<r_{\mathrm{m}}, \Delta r_{1}=r_{1}-r_{\mathrm{m}}<0, \quad f^{\prime}\left(r_{1}\right)=4 \pi r_{1}\left(3 P_{1} r_{1}+4 \sigma\right)=4 \pi r_{1}\left(3 P_{1} \Delta r_{1}\right)>0$. Therefore, $F\left(r_{1}\right)$ is a minimum and the equilibrium of an air bubble of radius $r_{1}$ is stable.

For $r_{2}>r_{\mathrm{m}}, \Delta r_{2}=r_{2}-r_{\mathrm{m}}>0, \quad f^{\prime}\left(r_{2}\right)=4 \pi r_{2}\left(3 P_{1} r_{2}+4 \sigma\right)=4 \pi r_{2}\left(3 P_{1} \Delta r_{2}\right)<0$. Therefore, $F\left(r_{2}\right)$ is a maximum and the equilibrium of an air bubble of radius $r_{2}$ is unstable.

3) If $P_{1}=P_{1}^{*}$, we got $F^{\prime}\left(r_{*}\right)=0$ and $F^{\prime \prime}\left(r_{*}\right)=0$. Therefore, $F\left(r_{*}\right)$ reaches its inflection point (Figure 2), leading the equilibrium of the bubble of $r_{*}$ is unstable.

4) When $P_{1}<P_{1}^{*}$, a gas bubble could not be at any equilibrium.

Every one of bubbles has its own $n R T$, also its own $P_{1}^{*}$, being called its Blake threshold pressure, and its $r_{*}$, or Blake critical radius [20] [21].

\section{Two Types of Cavitation}

\subsection{First Type of Cavitation: Growth of Pre-Existent Air Bubbles in Conduits}

Suppose that along with the decreasing of $P_{1}$ a bubble with $n$ mole air in a conduit of radius $r_{\mathrm{c}}$ enlarges stably. If its Blake radius $r_{*}>r_{\mathrm{c}}$ (or based on $\left.r_{*}=\frac{3}{2} \sqrt{\frac{n R T}{2 \pi \sigma}}, n R T=\frac{8 \pi \sigma}{9} r_{*}^{2}>\frac{8 \pi \sigma}{9} r_{\mathrm{c}}^{2}\right)$, before its exploding at $P_{1}^{*}=-4 \sigma / 3 r_{*}$, it has become long shaped, leading the bubble only to expand and lengthen gradually. This is the first way of cavitation. Only if $r_{*}<r_{\mathrm{c}}$, or $n R T<\frac{8 \pi \sigma}{9} r_{\mathrm{c}}^{2}$, can it explode at its $P_{1}=P_{1}^{*}$ to form a long bubble, and to lengthen gradually. This is the second way of cavitation, or the way of expanding-exploding, becoming a long bubble-lengthening gradually. Therefore, how a bubble develops depends on which of $r_{*}$ and $r_{c}$ is larger, or on which of $n R T$ and $\frac{8 \pi \sigma}{9} r_{\mathrm{c}}^{2}$ is larger. Thus, for a pre-existent air bubble the boundary of above two ways of cavitation is its $r_{*}=r_{\mathrm{c}}$ at $P_{1}^{*}=P_{\mathrm{lc}}^{*}=-4 \sigma / 3 r_{\mathrm{c}}$. The action of these two 
ways of cavitation all are the same with the first two ways of cavitation by air seeding [15] except of forming isolated embolized conduits without any reservoir.

\subsection{Second Type of Cavitation: Air Seeding}

When an air seed is sucked into a conduit of radius $r_{\mathrm{c}}$ from atmosphere through a pore of radius $r_{\mathrm{p}}$ in pit membrane, its initial radius equals $r_{\mathrm{p}}$ and initial gas pressure $P=P_{0}$ [22]. In the range of pressure $-2 P_{0}<P_{1}<P_{0}$ its radius should be $r_{0}^{\prime}, r_{0}$ or $r_{1}$ [17]. As $P_{1}$ decreases, it will develop like the growth of a pre-existent air bubble in a conduit, presenting the first or second ways of cavitation but with air reservoirs [15].

If a seed enters a conduit of radius $r_{\mathrm{c}}$ through a pore of radius $r_{\mathrm{pc}}$ in the conduit wall from atmosphere and will break up at $P_{\mathrm{lc}}^{*}=-4 \sigma / 3 r_{\mathrm{c}}$ with $r_{*}=r_{\mathrm{c}}$, there is a relationship $n R T=\frac{8 \pi \sigma}{9} r_{*}^{2}=\frac{8 \pi \sigma}{9} r_{\mathrm{c}}^{2}=P_{\mathrm{o}} \times \frac{4 \pi r_{\mathrm{pc}}^{3}}{3}$. Therefore,

$$
r_{\mathrm{pc}}=\left(2 \sigma r_{\mathrm{c}}^{2} / 3 P_{\mathrm{o}}\right)^{1 / 3}
$$

From $P_{1}=P_{\mathrm{o}}+P_{1}^{\prime}$ and $P_{1}^{\prime}=-2 \sigma / r_{\mathrm{pc}} \quad$ [22], the pressure $P_{1}$ at which the seed enters the conduit is

$$
P_{\mathrm{l}}=P_{\mathrm{lc}}=P_{\mathrm{o}}-2 \sigma / r_{\mathrm{pc}}=P_{\mathrm{o}}-\left(\frac{2 \sigma}{r_{\mathrm{c}}}\right)^{2 / 3}\left(3 P_{\mathrm{o}}\right)^{1 / 3}
$$

However, at the moment the radius of the seed reaches $r_{\mathrm{c}}$. Then, it should become a long shaped bubble. Thus, the exploding event might disappear.

Using formulas (9) and (10), and combining the results of the articles [15] [17] the following conclusions are obtained.

1) In the range of $P_{\mathrm{lc}} \leq P_{1}<P_{\mathrm{o}}$ and $r_{\mathrm{p}} \geq r_{\mathrm{pc}}$, the first way of cavitation will form.

2) In the range of $-2 P_{\mathrm{o}}<P_{1}<P_{\mathrm{lc}}$ and $0.487 \mu \mathrm{m}<r_{\mathrm{p}}<r_{\mathrm{pc}}$ the second way of cavitation will take place.

3) In $P_{1} \leq-2 P_{\mathrm{o}}$ and $r_{\mathrm{p}} \leq 0.487 \mu \mathrm{m}$, soon after an air seed is sucked into a conduit, as its radius is $r_{2}$ it will explode immediately and the conduit will be filled with the seed air instantly, presenting the third way of cavitation.

The experiments [8] [9] show that as primary xylem conduits were directly connected to air-filled spaces within the pith, inter-conduit air seeding was the primary mechanism. Thus, $P_{\mathrm{o}}$ in $P_{\mathrm{o}} \times \frac{4 \pi r_{\mathrm{pc}}^{3}}{3}=n R T$ should be replaced by internal air pressure $P_{\mathrm{a}}$, causing some data to be recalculated.

\section{Relationship of the Two Types of Cavitation}

For the development of air seeds, Table 1 gives the values of radii (in bold) of some seeds, which are just sucked into conduits, and their corresponding pressures $P_{1}$ (in bold). Also the values of corresponding $n R T, r_{0}, r_{*}$ and $P_{1}^{*}$ of the seeds of radii $r_{0}^{\prime}, r_{0}$ and $r_{1}$. For a seed of radius $r_{1}$ (or $r_{2}$ ), using the 
formula 8 the corresponding $r_{2}$ (or $r_{1}$ ) can be calculated. Note the two states of the bubble of radius $r_{1}$ and $r_{2}$ are at the same water pressure $P_{1}$.

If a seed of radius $r=r_{\mathrm{pc}}$ at $P_{1}=P_{\mathrm{lc}}$ enters a conduit of radius $r_{\mathrm{c}}=6.501 \mu \mathrm{m}$, from formulas 9 and 10, we got $r_{\mathrm{pc}}=2.740 \mu \mathrm{m}$ and $P_{\mathrm{lc}}=0.04672 \mathrm{MPa} \quad$ (Table 1 line 2). In the range of $P_{\mathrm{lc}} \leq P_{1}<P_{0}$ the bubbles will expand gradually (Table 1 line 1 and 2), presenting the first way of cavitation. In the range of $-2 P_{\mathrm{o}}<P_{1}<P_{\mathrm{lc}}$, the bubbles of $r_{0}^{\prime}, r_{0}$ and $r_{1}$ will expand to their respective $r_{*}$, presenting the second way of cavitation (Table 1 line $3 \rightarrow 5$ ). For the seed of radius $r_{1}$ during the dropping of $P_{1}$ it will break up at $P_{1}^{*}$ with $r=r_{*}$ before its radius reaches $r_{2}$. Thus, in the range of $-2 P_{0}<P_{1}<0$ the bubble of radius $r_{2}$ in the parentheses does not exist (Table 1 line 5). When $P_{1} \leq-2 P_{0}$ the air seeds of radius $r \leq 0.4867 \mu \mathrm{m}$ will explode soon after their entering, presenting the third way of cavitation (Table 1 lines $6 \rightarrow 9$ ).

All air seeds of radius $r_{0}^{\prime}, r_{0}$, or $r_{1}$ (lines $1 \rightarrow 5$ ) by air seeding in Table 1 can also be regarded as pre-existent air bubbles. Occasionally the air seeds of radii $r_{2}$ by air seeding can shrink to their corresponding $r_{1}$. Thus, for this kind of bubbles of radii $r_{1}$ (lines $7 \rightarrow 9$ ), during the decreasing of $P_{1}$, they will expand and burst at their $P_{1}^{*}$ with radii $r_{*}$. Thus, all bubbles of radii $r_{0}^{\prime}, r_{0}$, or $r_{1}$, no matter how they formed before, can be regarded as pre-existent, including the bubble of radius $r_{\mathrm{o}}=0.2810 \mu \mathrm{m}$ in line 6. For a conduit of $r_{\mathrm{c}}=6.500 \mu \mathrm{m}$ as long as $P_{1}<P_{\mathrm{lc}} \quad\left(P_{\mathrm{lc}}=0.04671 \mathrm{MPa}\right)$ during $P_{1}$ decreasing, except of the bubbles in the first two lines in Table 1, all other bubbles can develop from $r_{0}^{\prime}, r_{0}$ or $r_{1}$ to $r_{*}$, presenting the second way of cavitation.

This indicates that in the range of $P_{1} \leq-2 P_{0}$, for air seeding the third way of

Table 1. Development of air bubbles in a conduit of radius $r_{\mathrm{c}}=6.500 \mu \mathrm{m}$. After an air seed of $r_{0}^{\prime}, r_{0}, r_{1}$ or $r_{2}$ (in bold) with $n$ mole air at $P=P_{0}$ enters the conduit at pressure $P_{1}$ (in bold), three ways of cavitation will take place (lines $1 \rightarrow 2$, first way; lines $3 \rightarrow 5$, second way and lines $6 \rightarrow 9$, third way). On the other hand all the bubbles in Table 1 can be regarded as pre-existent. During the decreasing of $P_{1}$ they will expand. The bubbles of radius $r_{*}>r_{c}$ in lines $1 \rightarrow 2$ will grow gradually. The others will expand to their respective radii $r_{*}$ at $P_{1}^{*}$ to explode. The bubbles with the values in parentheses can't exist. $n R T / 10^{-12} \mathrm{~J}, \quad r / \mu \mathrm{m}, P_{1} / \mathrm{MPa}$.

\begin{tabular}{ccccccccc}
\hline & $n R T$ & $P_{1}$ & $r_{0}^{\prime}$ & $r_{0}$ & $r_{1}$ & $r_{2}$ & $r_{*}$ & $P_{1}^{*}$ \\
\hline 1 & 52.36 & $\mathbf{0 . 0 7 0 8 0}$ & $\mathbf{5 . 0 0 0}$ & $(9.253)$ & & & $(16.03)$ & -0.00607 \\
2 & 8.617 & $\mathbf{0 . 0 4 6 7 2}$ & $\mathbf{2 . 7 4 0}$ & 3.754 & & & $(6.501)$ & -0.01497 \\
3 & 3.351 & $\mathbf{0 . 0 2 7 0 0}$ & $\mathbf{2 . 0 0 0}$ & 2.341 & & & 4.054 & -0.02401 \\
4 & 1.304 & $\mathbf{0}$ & & $\mathbf{1 . 4 6 0}$ & & & 2.529 & -0.03849 \\
5 & 0.4190 & $-\mathbf{0 . 0 4 6 0 0}$ & & 0.8276 & $\mathbf{1 . 0 0 0}$ & $(2.919)$ & 1.433 & -0.06790 \\
6 & 0.04828 & $-\mathbf{0 . 2 0 0 0}$ & & 0.2810 & $\mathbf{0 . 4 8 6 7}$ & $\mathbf{0 . 4 8 6 7}$ & $\mathbf{0 . 4 8 6 7}$ & -0.2000 \\
7 & 0.04349 & $-\mathbf{0 . 2 1 0 6}$ & & 0.2667 & 0.4541 & $\mathbf{0 . 4 7 0 0}$ & 0.4619 & -0.2107 \\
8 & 0.002058 & $-\mathbf{0 . 7 5 8 8}$ & & 0.05801 & 0.07392 & $\mathbf{0 . 1 7 0 0}$ & 0.1005 & -0.9687 \\
9 & 0.0004189 & $-\mathbf{1 . 3 6 0}$ & & 0.02617 & 0.03104 & $\mathbf{0 . 1 0 0 0}$ & 0.04533 & -2.147 \\
\hline
\end{tabular}


cavitation will occur; but for pre-existent air bubbles of radius $r \leq 0.4867 \mu \mathrm{m}$ the second way will take place.

From Table 1 we can see that the more the amount $n R T$ of a bubble, the larger its Blake critical radius $r_{*}$ and the higher its Blake threshold pressure. This means that a bubble with more $n R T$ is prone to burst at higher pressure and only the nanobubbles with a small amount of air can exist steadily in larger ranges of water pressures. For example, in Table 1 at $P_{1}=0$ all bubbles of radii $r_{0} \geq 0.2810 \mu \mathrm{m}$ would burst at $P_{1}^{*} \geq-0.2000 \mathrm{MPa}$ with $r_{*} \geq 0.4867 \mu \mathrm{m}$. But in its last line, a bubble of radius $r_{0}=26.17 \mathrm{~nm}$ at $P_{1}=0$ will break at $P_{1}^{*}=-2.147 \mathrm{MPa}$ with the radius $r_{*}=45.33 \mathrm{~nm}$. Thus, in the large range of $P_{1}>-2.147 \mathrm{MPa}$ the bubble can exist temporarily steadily.

The scale bars shown in the figures of the article [11] is $50 \mu \mathrm{m}$. The nanobubbles, the radius of which was shorter than $0.4867 \mu \mathrm{m}$ (Table 1. line 6), might not be seen under light microscope before their bursting. Thus, the first type of cavitation proposed by Ponomarenko et al. [11] might be caused by nanobubbles.

There are insoluble surfactants in xylem sap. Because of surfactants effect, the values of $\sigma$ may decreases. From $P_{1}^{*}=-\frac{8 \sigma}{9} \sqrt{\frac{2 \pi \sigma}{n R T}}$ we can see that the smaller the $\sigma$, the smaller the absolute value of $P_{1}^{*}$, meaning that at higher water pressure an air bubble will burst and a cavitation event will occur easily. Thus, the values in Table 1 should be recalculated.

\section{Conclusions}

For the equation of bubble balance, using Shenjin formula, which is simpler than the Caldan's, this paper gets its analytic solutions. The stability of equilibrium of air bubbles was made by the way different from the previous in the article [17]. Two types of cavitation are analyzed further. For the first type of cavitation two ways can occur, which are the same with the first two ways of air seeding except of air reservoirs. Moreover, for the first way of the two types, the range of water pressures is the same. For the second way of the first type the range of water pressures is much wider, or the pressure range equals the pressure sum of the second and third ways of air seeding.

Through the specific data the relationship between the two types is given.

\section{Acknowledgements}

This work was financially supported by the National Natural Science Foundation of China (\#30270343).

\section{Conflicts of Interest}

None declared.

\section{References}

[1] Tyree, M.T. (1997) The Cohesion-Tension Theory of Sap Ascent: Current Contro- 
versies. Journal of Experimental Botany, 48, R1753-R1765.

https://doi.org/10.1093/jxb/48.10.1753

[2] Tyree, M.T. and Sperry, J.S. (1989) Vulnerability of Xylem to Cavitation and Embolism. Annual Review of Plant Physiology and Plant Molecular Biology, 40, R19-R38. https://doi.org/10.1146/annurev.pp.40.060189.000315

[3] Jackson, G.E. and Grace, J. (1996) Field Measurement of Xylem Cavitation: Are Acoustic Emission Useful? Journal of Experimental Botany, 47, R1643-R1650. https://doi.org/10.1093/jxb/47.11.1643

[4] Cochard, H., Cruiziat, P. and Tyree, M.T. (1992) Use of Positive Pressures to Establish Vulnerability Curves. Further Support for the Air-Seeding Hypothesis and Implications for Pressure-Volume Analysis. Plant Physiology, 100, 205-209. https://doi.org/10.1104/pp.100.1.205

[5] Zimmermann, M.H. (1983) Xylem structure and the Ascent of sap. Springer, Berlin, 39-47. https://doi.org/10.1007/978-3-662-22627-8

[6] Jansen, S., Klepsch, M., Li, S., Kotowska, M.M., Schiele, S., Zhang, Y. and Schenk, H.J. (2018) Challenges in Understanding Air-Seeding in Angiosperm Xylem. Acta Horticulturae, 1222, 13-20. https://doi.org/10.17660/ActaHortic.2018.1222.3

[7] Rockwell, F.E., Wheeler, J.K. and Holbrook, N.M. (2014) Cavitation and Its Discontents: Opportunities for Resolving Current Controversies. Plant Physiology, 164, 1649-1660. https://doi.org/10.1104/pp.113.233817

[8] Brodersen, C.R., McElrone, A.J., Choat, B., Lee, E.F., Shackel, K.A. and Matthews, M.A. (2013) In Vivo Visualizations of Drought-Induced Embolism Spread in Vitis vinifera. Plant Physiology, 161, 1820-1829. https://doi.org/10.1104/pp.112.212712

[9] Choat, B., Brodersen, C.R. and McElrone, A.J. (2015) Synchrotron X-Ray Microtomography of Xylem Embolism in Sequoia Sempervirens Saplings during Cycles of Drought and Recovery. New Phytologist, 205, 1095-1105.

https://doi.org/10.1111/nph.13110

[10] Mayr, S., Kartusch, B. and Kikuta, S. (2014) Evidence for Air-Seeding: Watching the Formation of Embolism in Conifer Xylem. Journal of Plant Hydraulics, 1, e004. https://doi.org/10.20870/jph.2014.e004

[11] Ponomarenko, A., Vincent, O., Pietriga, A., Cochard, H., Badel, E. and Marmottant, P. (2014) Ultrasonic Emissions Reveal Individual Cavitation Bubbles in Water-Stressed Wood. Journal of the Royal Society Interface, 11, Article ID: 20140480. https://doi.org/10.1098/rsif.2014.0480

[12] Kanduc, M., Schneck, E., Loche, P., Jansen, S., Schenk, H.J. and Netz, R.R. (2020) Cavitation in Lipid Bilayers Poses Strict Negative Pressure Stability Limit in Biological Liquids. Proceedings of the National Academy of Sciences of the United States of America, 117, 10733-10739. https://doi.org/10.1073/pnas.1917195117

[13] Plavcová, L., Jansen, S., Klepsch, M. and Hacke, U.G. (2013) Nobody’s Perfect: Can Irregularities in Pit Structure Influence Vulnerability to Cavitation? Frontiers in Plant Science, 4, 453-458. https://doi.org/10.3389/fpls.2013.00453

[14] Schenk, H.J., Steppe, K. and Jansen, S. (2015) Nanobubbles: A New Paradigm for Air-Seeding in Xylem. Trends in Plant Science, 20, 199-205. https://doi.org/10.1016/j.tplants.2015.01.008

[15] Shen, F.Y., Wang, Y.S., Cheng, Y.X. and Zhang, L. (2012) Three Types of Cavitation Caused by Air Seeding. Tree Physiology, 32, 1413-1419. https://doi.org/10.1093/treephys/tps089

[16] Shen, F.Y., Gao, R.F., Liu, W.J. and Zhang, W.J. (2002) Physical Analysis of the Process of Cavitation in Xylem Sap. Tree Physiology, 22, 655-659. 
https://doi.org/10.1093/treephys/22.9.655

[17] Shen, F.Y., Liu, W.J., Gao, R.F. and Huo, H. (2003) A Careful Physical Analysis of Gas Bubble Dynamics in Xylem. Journal of Theoretical Biology, 225, 229-233. https://doi.org/10.1016/S0022-5193(03)00241-8

[18] Konrad, W., Katul, G., Roth-Nebelsick, A. and Jensen, K.H. (2019) Xylem Functioning, Dysfunction and Repair: A Physical Perspective and Implications for Phloem Transport. Tree Physiology, 39, 243-261. https://doi.org/10.1093/treephys/tpy097

[19] Fan, S. (1989) A New Extracting Formula and a New Distinguished Means on the One Valuable Cubic Equation. Natural Science Journal of Hainan Teachers College, 2, 91-98. (In Chinese)

[20] Christopher, E.B. (1995) Cavitation and Bubble Dynamics. Oxford University Press, New York.

[21] Harkin, A., Nadim, A. and Kaper, T.J. (1999) On Acoustic Cavitation of Slightly Subcritical Bubbles. Physics of Fluids, 11, 274-287. https://doi.org/10.1063/1.869878

[22] Sperry, J.S. and Tyree, M.T. (1988) Mechanism of Water Stress-Induced Xylem Embolism. Plant Physiology, 88, 581-587. https://doi.org/10.1104/pp.88.3.581

\section{Nomenclature}

$P_{1}$ : absolute water pressure in a conduit

$r$. radius of a spherical air bubble

$r_{0}$ : radius of a spherical air bubble at $P_{1}=0$

$r_{0}^{\prime}$ : radius of a spherical air bubble at $P_{1}>0$

$V_{\mathrm{g}}$ : bubble volume

$R$ : gas constant

$T$ : absolute temperature

$n$ : molar number of air in a bubble

$P_{\mathrm{o}}$ : atmospheric pressure

$P$ : gas pressure of a bubble

$P_{\mathrm{g}}:$ gas pressure of an air bubble in equilibrium

$\sigma:$ surface tension of xylem water

$P_{1}^{*}$ : Blake threshold pressure of an air bubble

$r_{*}$, Blake critical radius of an air bubble

$r_{\mathrm{c}}$ : radius of a conduit

$r_{\mathrm{p}}$ : radius of a pore in pit membrane

$P_{\mathrm{lc}}^{*}$ : absolute water pressure at which an air bubble of radius $r_{*}=r_{\mathrm{c}}$ will burst

$r_{\mathrm{pc}}$ : radius of the pore through which an air seed enters a conduit of radius $r_{\mathrm{c}}$ and will burst at $P_{\mathrm{lc}}^{*}$

$P_{\mathrm{lc}}$ : absolute water pressure at which an air seed enters a conduit of radius $r_{\mathrm{c}}$ and will burst at $P_{\mathrm{lc}}^{*}$

$F(r)$ : Helmholtz function

$A$ : gas/water interface 